\title{
Atenção online de artigos do periódico Ciência e Agrotecnologia
}

\begin{abstract}
Nivaldo Calixto Ribeiro
Resumo: As métricas são fundamentais para qualquer periódico de qualidade. Com elas, é possível analisar as citações e a repercussão de seus artigos em diversas fontes de informação na internet. A almetria analisa as métricas de um documento por meio da quantidade de compartilhamentos e outros atributos em mídias sociais. Nesta abordagem, esta pesquisa tem como objetivo analisar a atenção online de artigos da Ciência e Agrotecnologia na web social. As investigações incidem sobre a análise de como os leitores desse periódico mencionam os seus artigos em plataformas de mídias sociais. Como recurso metodológico, foi adotada a altmetria para verificar métricas relacionadas à atenção online sobre os artigos desse periódico, utilizando-se para a coleta de dados a ferramenta Altmetric.com. Foram identificadas apenas 43 menções de 36 artigos desse periódico. Foram realizadas 29 menções em plataformas de mídias sociais, $10 \mathrm{em}$ sites de políticas/patentes, três na Wikipedia e um em vídeo. A ferramenta apontou que 35 delas obtiveram ao menos o Almetric Attetion Score equivalente a 1. A plataforma de mídia social com maior índice de menções foi o Twitter. Observou-se que há uma tímida atenção na web social, bem como poucas menções de publicações da Ciência e Agrotecnologia.
\end{abstract}

Palavras-Chave: Comunicação científica; Altmetria; Ciência e Agrotecnologia; Periódico.

Abstract: Metrics are essential for any quality journal. With them it is possible to analyze the citations and the repercussion of your articles in several sources of information on the internet. Almetria analyzes the metrics of a document through the amount of shares and other attributes on social media. In this approach, this research aims to analyze the online attention of "Science \& Agrotechnology" articles on the social web. The investigations focus on the analysis of how the readers of this journal mention their articles on social media platforms. As a methodological resource, altmetry was adopted to verify metrics related to online attention on the articles in this journal, using the Altmetric.com tool for data collection. It was identified that there were 49 screened outputs of articles from "Science and Agrotechnology". Only 43 mentions of 36 articles from this journal were identified. 29 mentions were made on social media platforms, 10 on policy / patent sites, 3 on Wikipedia and one on video. The tool pointed out that 35 of them obtained at least the Almetric Attetion Score equivalent to 1. The social media platform with the highest index of mentions was Twitter. He noted that there is a shy attention on the social web, as well as few mentions of publications from "Science and Agrotechnology".

Key words: Scientific communication; Altmetry; Science and Agrotechnology Periodic. 


\section{INTRODUÇÃO}

A comunicação e a divulgação dos resultados de pesquisa têm se tornado um grande desafio para os pesquisadores e instituições de fomento, compreendendo que a produção de pesquisa deve gerar impacto e retornos tangíveis e perceptíveis para a comunidade em geral, empresas e órgãos públicos. É incomum que esse público consulte diretamente a literatura científica, devido a inúmeros fatores que dificultam o acesso a esse tipo de informação, o que tem como consequência a baixa aceitação e pouca disseminação de resultados de pesquisas fora da academia.

As mídias sociais, devido às suas especificidades e alta capacidade de disseminação da informação, mostram-se como um relevante recurso para que os pesquisadores e instituições de fomento à pesquisa compartilhem suas descobertas com a comunidade, de forma a despertar o interesse desse público pela ciência.

Nas atividades acadêmicas, como em todos os níveis e segmentos da sociedade, as mídias sociais estão muito latentes (OLIVEIRA, 2012). Aproveitando-se desse fenômeno, os editores e os responsáveis pelas revistas científicas passaram a usá-las como plataforma de comunicação para informar o seu público a respeito dos últimos artigos divulgados, possibilitando a interação mais rápida e imediata com seus leitores, ampliando a visibilidade, o alcance e a disseminação de suas publicações.

A presença em mídias sociais de periódicos acadêmicos, em grande medida, pode, portanto, facilitar a comunicação e a divulgação de resultados de pesquisa para um público mais amplo (ZHENG et al., 2019). Consequentemente, as mídias sociais podem gerar inúmeras métricas de impacto de pesquisa, de forma mais célere, por meio da altmetria.

Esse recurso metodológico tem expandido a visão da comunidade acadêmica a respeito de como os procedimentos e os fatores de impacto tradicionais das publicações têm se apresentado, estendendo as análises e métricas de citações às informações geradas nas mídias sociais, por meio de armazenamento, de links, de marcadores e de comentários (MILOJEVIĆ; SUGIMOTO, 2012). O uso apenas do fator de impacto pode subestimar a importância de periódicos menores e especializados. Nenhuma métrica pode servir como uma avaliação perfeita do valor de uma revista (VRABEL, 2019). 
No contexto apresentado, englobando a comunicação científica e a aplicação de métricas alternativas, a investigação em questão tem como objetivo analisar a atenção online de artigos da Ciência e Agrotecnologia na web social, periódico científico financiado pela Universidade Federal de Lavras (UFLA) e pela Fundação de Amparo à Pesquisa do Estado de Minas Gerais (Fapemig), editado bimensalmente pela Editora UFLA e com o apoio das Pró-reitorias de Pesquisa e de Graduação dessa mesma universidade. Desde a alteração do título da Ciência e Agrotecnologia, anteriormente, Ciência e Prática, no ano de 1996, foram publicados 96 volumes, com artigos científicos nas áreas de ciências vegetais e animais, incluindo agronomia, ciência e tecnologia de alimentos, economia e administração do agronegócio, engenharia rural, veterinária e zootecnia (UNIVERSIDADE FEDERAL DE LAVRAS, 2020).

O periódico é citado em fontes indexadoras, como o Sistema Internacional de Informação para as Ciências Agrárias e Tecnológicas (AGRIS); a Base de Dados da Agricultura Brasileira (AGROBASE); o Centro Internacional de Pesquisa Científica (CIRS); o Web of Science - Thomson Reuters (WoS); o Sistema Regional de Informação em Linha para Revistas Científicas da América Latina, Caribe, Espanã e Portugal (LATINDEX); a Literatura Latino-Americana e do Caribe em Ciências da Saúde (LILACS); o Scientific Eletronic Library Online (SciELO); o Scopus da Elsevier e outros (UNIVERSIDADE FEDERAL DE LAVRAS, 2020).

De acordo com a Plataforma Sucupira, da Coordenação de Aperfeiçoamento de Pessoal de Nível Superior (CAPES, 2020), no triênio 2013-2016, a Ciência e Agrotecnologia foi classificada pelo Qualis em diversas áreas, com destaque para a classificação A2 para a área de ciências agrárias e B1 para as áreas de administração pública e de empresas, ciências contábeis e turismo; ciência de alimentos; ciências ambientais; enfermagem; engenharias e zootecnia / recursos pesqueiros.

Como prática príncipua de uma investigação, busca-se responder questões relacionadas ao objeto de estudo. Assim, adotando as publicações da Ciência e Agrotecnologia como pontos centrais para este estudo, procura-se identificar: quantos foram mencionados em mídias sociais? Quais foram as plataformas de mídias sociais mais utilizadas por seus leitores? Esse periódico científico dissemina suas descobertas de pesquisa e se comunica com seus leitores por meio das mídias sociais, visando, assim, popularizar os resultados de suas publicações? 


\section{AVALIAÇÃO CIENTÍFICA POR MEIO DE INDICADORES ALTMÉTRICOS}

Computar citações pode ser considerado como um formato padrão para mensurar o impacto de uma pesquisa e/ou pesquisador. Na perspectiva de Gouveia (2016), no campo da comunicação científica, o fator de impacto demonstra o que ocorreu anteriormente, sem apresentar garantias futuras. Nesse viés, Oliveira (2019) expõe que a divulgação da ciência tem passado por grandes transformações, que vão além das possibilidades abertas, favorecidas pelas tecnologias digitais da era da informação.

Observa-se uma transição das atividades acadêmicas e científicas para a internet, o que tem modificado a forma de analisar os impactos de uma publicação, devido aos mais diversos meios de divulgação de resultados de pesquisa, principalmente, via plataformas de redes sociais (SHEKHAWAT; CHAUHAN, 2019). De acordo com os autores, os cientistas passaram a utilizar as mídias sociais para diversas finalidades no percurso de seus projetos de pesquisas e na sua rotina acadêmica. Para Varella (2019), a divulgação científica nas plataformas de mídias sociais tem se tornado um grande desafio para editores de periódico e para a comunicação científica.

É justamente nesse contexto que a altmetria vem ganhando reconhecimento como métrica alternativa para avaliar publicações com dados de mídias sociais, complementando as possibilidades de avaliação do impacto de uma publicação (BORBA; ALVAREZ; CAREGNATO, 2019; COSTA; OLIVEIRA; ARAÚJO, 2019). Abordagens altmétricas passaram a serem vistas no ecossistema científico, após a publicação do Manifesto Altmetrics, publicado por Jason Priem, em 2010 (PRIEM et al., 2010). Segundo Gouveia (2019), esse foi o "marco inicial para definição do campo e sua separação dos estudos webométricos".

Trabalhos de pesquisa anteriores também investigaram a altmetria e seu impacto em periódicos acadêmicos, bem como em produtos de pesquisa. As métricas alternativas alavancam as mídias sociais e outras plataformas online no intuito de disponibilizar repercussões imediatas de alcance de pesquisas científicas entre os mais variados públicos (ROSEN KRANTZ; AYOOLA; SINGH, 2017). A seguir, são apresentadas publicações com objetos de estudo similares ao deste trabalho.

A investigação de Hayon, Tripathi e Stormont (2019) teve como objetivo quantificar a relação entre o número de menções no Twitter e o número de citações 
acadêmicas que publicações sobre urologia receberam. Foram analisados 213 artigos de sete periódicos sobre esse tema. Os artigos foram avaliados com técnicas bibliométricas baseadas em citações (Scopus, Google Scholar) e as menções do Twitter foram rastreadas usando o Altmetric. Os dados foram tratados por meio da ANOVA, que são modelos estatísticos nos quais a variância amostral é dividida em um composto de distintas variáveis. Os autores concluíram em suas análises que a maioria das publicações urológicas está sendo compartilhada no Twitter. O número de citações que uma publicação urológica recebe até 3 anos após o lançamento está positivamente associado ao número de menções que ela tem no Twitter.

Em outra investigação, Rosenkrantz, Ayoola e Singh (2017) concluíram em seus estudos que, embora a atenção online para o conteúdo de periódicos de radiologia seja baixa, métricas alternativas exibem tendências exclusivas, particularmente para artigos não-clínicos, e podem fornecer uma medida complementar do impacto da pesquisa em radiologia, em comparação com as métricas tradicionais de citações. Os autores compararam as métricas tradicionais de impacto e altmétrico dos artigos de revistas populares de radiologia. No total, foram 892 pesquisas originais publicadas em 2013 nas edições da Academic Radiology, do American Journal of Roentgenology, do Journal of the American College of Radiology e do Radiology. As citações tradicionais dos artigos desses periódicos foram obtidas por meio da Web of Science. A Altmetric Attention Score de cada artigo, representando menções em plataformas mídias sociais online, foi obtida por meio da ferramenta Altmetric.com.

O artigo de Araújo (2015) reflete sobre os impactos e possíveis relações entre dados altmétricos e citações, assim, contribuem com a discussão, tendo como análise empírica a Revista DataGramaZero. Os dados altmétricos foram levantados por meio de parametrizações de APIs (Facebook e Twitter) e, para a coleta das citações, recorreu-se ao Publish or Perish com dados do Google.Scholar. O autor observou que os artigos antigos têm maior índice de citações e artigos recentes apresentam mais dados altmétricos.

A literatura aqui apresentada mostra que a altmetria apresenta-se como uma possibilidade de análise das relações entre as mídias sociais e a produção científica. Ainda, infere-se que dados altmétricos indicam a atenção que os artigos recebem logo 
após a publicação e podem ser indícios de citações futuras entre outras possibilidades de informações que podem ser melhor exploradas por meio de métricas alternativas.

\section{PERCURSO METODOLÓGICO}

Em relação aos procedimentos metodológicos adotados, este estudo apresenta características qualitativas e quantitativas, podendo-se ser categorizado como exploratório. Está voltado para o levantamento, sistematização e análise de dados altmétricos, no intuito de identificar as relações estabelecidas entre documentos publicados pela Ciência e Agrotecnologia. O foco é medir menções em mídias sociais para os artigos que tenham um Digital Object Indentifer (DOI) ou outro identificador padrão.

No primeiro passo, foi utilizada a ferramenta Altmetric, devido ao seu foco na engenharia, no conhecimento e na ciência de dados, possibilitando a coleta e a análise da interação online em torno dos resultados de pesquisa. Essa ferramenta oferece recursos para auxiliar no monitoramento, na navegação, na pesquisa, no filtro e na avaliação de conversas em torno de uma pesquisa publicada, mencionada em blogs, mídias sociais e gestores de referências (Mendeley, CiteULike e o Zotero), de qualquer artigo com DOI ou Uniform Resource Identifier (URI). Além disso, fornece percepções detalhadas de atenção e contribui para ter uma visão mais rica do alcance e do impacto de uma pesquisa. É possível identificar quem foi comentado sobre sua pesquisa. Demonstra o impacto de seu trabalho para financiadores e comitês de avaliação. Monitora como uma pesquisa financiada foi recebida e disseminada, além de demonstrar onde ela está influenciando em alguma política pública (ALMETRIC, 2019). Como estratégia de busca da pesquisa no Altmetric, foi utilizada a expressão Ciência e Agrotecnologia, na opção de "título da fonte". Não houve uma delimitação temporal, visto que pretendeu-se analisar todas as menções a respeito da Ciência e Agrotecnologia nas mídias sociais e outras plataformas online em qualquer ocasião.

O próximo passo foi gerar, no Altmetric, os dados relacionados à quantidade de menções dos artigos da Ciência e Agrotecnologia, especificando a quantidade de citações em plataformas de mídias sociais e demais plataformas web, onde foram mencionadas e outros indicadores. Em seguida, foi gerado um ranking com os 10 
artigos de melhor índice Almetric Attetion Score e outros critérios, devidamente explanado na próxima sessão. O índice Almetric Attetion Score refere-se uma pontuação que é atribuída a uma publicação ou conjunto de dados, considerando a contagem ponderada por meio de um algorítmo de todas as menções que essa ferramenta rastreia para uma saída de pesquisa individual, apontando um indicador da quantidade, do engajamento e do alcance da atenção online que um item recebeu.

Por fim, foi realizada uma análise qualitativa das menções dos artigos ranqueados, observando as principais características e os contextos abordados pelos leitores e usuários das plataformas de mídias sociais.

\section{RESULTADOS E DISCUSSÃO}

Com o propósito de responder às questões desta pesquisa, os dados foram coletados e exportados para uma planilha em formato excel para posterior análise. Além disso, a interface da ferramenta Altmetric, com os resultados da estratégia de busca, fora verificada durante as análises, assim, serão expostas a seguir as principais observações:

1. Foi identificado que houve 49 saídas rastreadas de artigos da Ciência $e$ Agrotecnologia, mas foram identificadas apenas 43 menções de 36 artigos desse periódico. Foram realizadas 29 menções em plataformas de mídias sociais, 10 em sites de políticas/patentes, 3 na Wikipedia e um em vídeo. A ferramenta apontou que 35 delas obtiveram ao menos o Almetric Attetion Score equivalente a um.

2. Segundo o Scielo Analytics (2019), até agosto de 2019, foram publicados 2.309 documentos da Ciência e Agrotecnologia. No Altmetric, foram identificados 49 artigos com saídas rastreadas, indicando que apenas 2,12\% dos artigos dessa publicação foram citados na web social.

3. Do total de menções em mídias sociais, 24 foram realizadas no Twitter, três no Facebook e duas no Google+. Para Shekhawat e Chauhan (2019), o Twitter tem uma base de usuários bem ampla e parece ser particularmente adequado para o compartilhamento de informações de pesquisa, pois fornece uma maneira fácil de compartilhar informações para os seguidores que, por sua vez, podem encaminhar novamente a outros usuários. 
4. Com relação aos sites de políticas/patentes, foram constatadas nove menções em patentes, que são um tipo de propriedade intelectual protegida por um inventor ou por um detentor e um documento relacionado a políticas públicas. $\mathrm{O}$ Altmetric acompanha uma ampla gama de documentos de menções direcionadas a políticas públicas, entendido como qualquer documento de regulamentação, orientação ou diretrizes de uma organização governamental ou não-governamental.

5. Houve três menções na Wikipedia, coletadas da seção de referências de artigos redigidos na língua inglesa, um deles trata sobre o zoneamento climático do estado do Paraná, Brasil; outro sobre o efeito da umidade e do período de armazenamento hermético na contaminação natural por fungos e na produção de micotoxinas em grãos de aveia e o terceiro aborda a produção de ácido acético, etanol e dos isômeros ópticos do ácido lático por linhagens de Lactobacillus isoladas de fermentações alcoólicas industriais. Houve apenas uma menção em um vídeo sobre alimentos ácidos, no canal "Detona Química"1, disponível na plataforma de vídeo do YouTube, em 12 de fevereiro de 2018, com 1.919 visualizações, em 10 de fevereiro de 2020. O canal propõe a apresentação da química de forma divertida, o que sugere a aproximação de conceitos científicos com uma linguagem mais próxima da população. A menção consta na descrição do vídeo, como fonte da pesquisa que auxiliou na construção do vídeo.

6. Ao efetuar a análise por demografia, percebeu-se que as menções oriundas do Twitter foram realizadas em perfis do Brasil, com 11 menções, Estados Unidos da América e Holanda, Venezuela e Bolívia com 1 menção. Além disso, em nove das menções nessa plataforma não foi possível identificar a origem.

7. Com relação ao Facebook, houve uma postagem realizadas da Austrália e outras duas não foi identificado o país de origem. Ainda houve um documento relacionado à política na Itália.

8. Por meio desta pesquisa, foi identificado que há 500 artigos da Ciência e Agrotecnologia salvos na biblioteca dos usuários do Mendeley, programa desktop e web produzido pela Elsevier para gerenciar e compartilhar

\footnotetext{
${ }^{1}$ DETONANDO QUÍMICA. Qual será o alimento mais ácido?. 2018. (3m47s). Disponível em: https://www.youtube.com/watch?v=FRLhOGJoBcw. Acesso em: 10 fev. 2020.
} 
documentos de pesquisa, descobrindo dados de pesquisa e de colaboração online. Shekhawat e Chauhan (2019) indicam que o Mendeley é supostamente utilizado para armazenar referências que os usuários já possam ter lido ou que pretendem ler futuramente. Os autores sugerem que as contagens de "leitores" de artigos nesse gerenciador de referência se assemelham às contagens de citações que representam o impacto de um artigo.

Em seguida, será apresentada uma análise dos artigos da Ciência $e$ Agrotecnologia mencionados em mídias sociais, com uma abordagem qualitativa. Os 10 artigos foram ranqueados, observando-se o Altmetric Attention Score, a incidência de vezes que o artigo foi salvo nas bibliotecas dos usuários do Mendeley, o número de menções no Twitter, seguido pelos demais indicadores citados no Quadro 2:

Quadro 2 - Artigos com melhores ranqueados

\begin{tabular}{|c|c|c|c|c|c|c|c|c|}
\hline \multicolumn{2}{|c|}{ Título da publicacão } & AS & $\mathbf{M}$ & $\mathbf{T}$ & $\mathbf{F}$ & $\mathbf{s}$ & D & $\mathbf{P}$ \\
\hline 1 & $\begin{array}{l}\text { Study of preparations of bee pollen extracts, antioxidant } \\
\text { and antibacterial activity }(2007) \\
\text { http } / / \text { dx.doi.org } / 10.1590 / \mathrm{S} 1413-70542007000600032\end{array}$ & 3 & 61 & - & - & - & 46 & 1 \\
\hline 2 & $\begin{array}{l}\text { Volatiles produced by interacting microorganisms } \\
\text { potentially useful for the control of plant pathogen } \\
(2010) \\
\text { http } / / \mathrm{dx} \text {.doi.org } / 10.1590 / \mathrm{S} 1413-70542010000300001\end{array}$ & 3 & 55 & - & - & 23 & 22 & 3 \\
\hline 3 & $\begin{array}{l}\text { Köppen, Thornthwaite and Camargo climate } \\
\text { classifications for climatic zoning in the State of Paraná, } \\
\text { Brazil (2016) } \\
\text { http://dx.doi.org/10.1590/1413-70542016404003916 }\end{array}$ & 3 & 42 & - & - & 26 & 9 & - \\
\hline 4 & $\begin{array}{l}\text { Efeito da umidade e do periodo de armazenamento } \\
\text { hermético na contaminação natural por fungos e a } \\
\text { produção de micotoxinas em grãos de aveia }(2006) \\
\text { http://dx.doi.org } / 10.1590 / \mathrm{S} 1413-70542006000100017\end{array}$ & 3 & 32 & - & - & - & 11 & - \\
\hline 5 & $\begin{array}{l}\text { The table egg: a review (2013) } \\
\mathrm{http} / / \mathrm{dx} \text {.doi.org/10.1590/S1413-70542013000200001 }\end{array}$ & 3 & 20 & 3 & - & 63 & 6 & - \\
\hline 6 & $\begin{array}{l}\text { Load-bearing capacity of a red-yellow latosol cultivated } \\
\text { with coffee plants subjected to different weed } \\
\text { managements (2013) } \\
\text { http://dx.doi.org/10.1590/S1413-70542013000200005 }\end{array}$ & 3 & 4 & - & - & 1 & 4 & 1 \\
\hline 7 & $\begin{array}{l}\text { Estabelecimento in vitro de estrelicia (Strelitzia reginae } \\
\text { Banks.) (2004) } \\
\text { http://dx.doi.org/10.1590/S1413-70542004000500009 }\end{array}$ & 3 & 4 & - & - & - & 5 & 1 \\
\hline 8 & $\begin{array}{l}\text { Germinação de sementes de café (Coffea arabica L.) } \\
\text { submetidas a diferentes concentrações e tempos de } \\
\text { embebição em celulase ( } 2013 \text { ) } \\
\text { http://dx.doi.org/10.1590/S1413-70542003000300009. }\end{array}$ & 3 & - & - & - & - & 1 & 3 \\
\hline 9 & $\begin{array}{l}\text { Data research of foodborne diseases outbreaks from } \\
\text { state of Paraná Brazil, in the period from } 1978 \text { to } 2000 \\
\mathrm{http} / / \mathrm{dx} \text {.doi.org } / 10.1590 / \mathrm{S} 1413-70542006000600016\end{array}$ & 1 & 74 & 1 & - & 2 & 4 & - \\
\hline
\end{tabular}

Legenda: $\mathrm{AS}=$ Altmetric Attetion Score; $\mathrm{M}=$ Mendeley; $\mathrm{T}=$ Twitter $; \mathrm{S}=$ Scopus;

$\mathrm{D}=$ Dimension

Fonte: Dados da pesquisa, 2020.

Por meio do Quadro 2, observou-se que os títulos melhores ranqueados apresentam 61, 55 e 20 menções no sistema gerenciador de referência Mendeley. Com o objetivo de contrastar o número de citações de cada um dos artigos melhores 
ranqueados, foi realizada uma consulta por título do artigo no Dimensions e identificou-se que, entre os artigos mais citados em mídias sociais, o que tem mais menções também é o mais citado nessa plataforma. O Dimensions é um sistema que reúne elementos, publicações, citações, métricas alternativas, ensaios clínicos, patentes e documentos de políticas com o propósito de disponibilizar uma plataforma que permite aos usuários encontrar e acessar informações relevantes de forma mais rápida e ampla, assim como resultados acadêmicos e de pesquisa, possibilitando insights para a definição de estratégia futura.

Retomando os resultados desta pesquisa, chama a atenção o $7^{\circ}$ artigo, que foi salvo 74 vezes em biblioteca dos usuários do Mendeley, o que pode prejulgar futuras citações. Ainda, observa-se que, dos sete artigos elencados, seis são publicações de documentos relacionados a políticas ou patentes. Outra observação importante é que, ao analisar o Altmetric Attetion Score, nota-se que a maioria alcança o indicador 3, o que pode ser considerado baixo e que, mesmo esses artigos sendo os mais citados, ainda assim, obtiveram um baixo impacto nas mídias sociais.

Ao analisar as postagens no Twitter, observou-se que, em 21 das menções, os usuários dessa rede postaram apenas o link de algum artigo da Ciência $e$ Agrotecnologia, com ou sem a citação do títulos.

Em uma das postagens, um usuário indica o título "Técnica de fistulação e canulação do rúmen em bovinos e ovinos" para esclarecer um diálogo virtual sobre fistulação de vacas. Segundo Santos (2019), essa técnica internacionalmente conhecida é constantemente alvo de crítica por parte dos defensores dos direitos dos animais. Frequentemente são lançadas petições para abolir essa técnica em pesquisas agropecuárias.

O título "Nutritional and microbiological quality of common beans (Phaseolus vulgaris L.) cooked with or without the use of soaking water", publicado em 2008, foi citado no Twitter, em 2014, seis anos após a sua publicação, para solucionar questões cotidianas como pode ser observado em "Já está na hora de desmitificar o feijão. Chega de crendice, se colocar de molho perde os nutrientes”. Nesse mesmo contexto, o título “The table egg: a review”, publicado em 2013, foi citado diversas vezes no mesmo ano de sua publicação, em perfis de países latino-americanos com comentários similares ao 
seguinte: “Este artículo hay q leerlo. importante para nuestra alimentación!”, também para solucionar questões cotidianas.

Apesar do baixo número de menções das publicações da Ciência $e$ Agrotecnologia, observa-se que as mídias sociais contribuem para uma aproximação da comunidade em geral em relação às publicações científicas, merecendo mais atenção dos editores de periódicos científicos a esse recurso.

\section{CONSIDERAÇÕES FINAIS}

A existência do perfil ou página da Ciência e Agrotecnologia foi consultada nas mídias sociais Twitter, Facebook e outras, entretanto, não se localizou. O site oficial do periódico e o cadastro em bases de dados onde fora indexado também foram consultados e não foram identificadas informações ou políticas de incentivo ao uso, comentários e compartilhamento de suas publicações em mídias sociais. Ainda assim, considerou-se relevante manter o andamento da pesquisa, visto que a presença na web social e citações a respeito da Ciência e Agrotecnologia nas mídias sociais independem da existência de perfil do periódico nas diversas plataformas existentes.

Com relação ao objetivo principal desta pesquisa, de analisar a atenção online de artigos da Ciência e Agrotecnologia em mídias sociais, observou-se que há uma tímida atenção na web social, bem como poucas menções de suas publicações. O que pode ser proporcionalmente invertido com relação às métricas tradicionais de fator de impacto.

Os dados altmétricos podem ser úteis como um auxílio para ampliar o impacto e a visibilidade das publicações do periódico, objeto deste estudo. Afirmar que há uma relação direta e proporcional entre as métricas, tradicionais e alternativas, não foi possível constatar. Entretanto, o registro significativo de artigos da Ciência $e$ Agrotecnologia na plataforma Dimensions e em outras fontes indexadoras pode indicar a possibilidade de futuras citações. É inquestionável a circulação de citações e, de certa forma, o impacto do periódico e o número de citações de suas publicações nos mostram a relevância desse título para as áreas de agrária e afins.

Recomenda-se para gestores, editores e equipe editorial de periódicos científicos um planejamento para o acompanhamento dessas novas métricas e o engajamento para atuação junto às mídias sociais. 
Apesar da baixa presença nas mídias sociais, pode-se afirmar que o Twitter tem se mostrado como a plataforma da web social com a qual os leitores da Ciência e Agrotecnologia tiveram mais envolvimento.

Por fim, pode-se dizer que é inquestionável que as mídias sociais têm o poder de ampliar a visibilidade e o alcance das pesquisas científicas realizadas, rompendo os muros da comunidade científica, chegando aos mais diversos públicos e popularizando, dessa forma, a ciência em si.

\section{AGRADECIMENTO}

"Os autores expressam agradecimento à Altmetric.com por fornecer os dados altmétricos deste estudo gratuitamente para fins de pesquisa".

\section{REFERÊNCIAS}

ALTMETRIC. Who's talking about your research?. 2020a. Disponível em: https://www.altmetric.com/. Acesso em: 10 jan. 2020.

ALTMETRIC. How is the Altmetric Attention Score calculated?. 2020b. Disponível em:

https://help.altmetric.com/support/solutions/articles/6000060969-how-is-the-altmetric-at tention-score-calculated-. Acesso em: 10 jan. 2020.

ARAÚJO, R. F. Da altmetria à análise de citações: uma análise da revista Datagramazero. DataGramaZero, Rio de Janeiro, v. 16, n.1, 2015. Disponível em: http://www.brapci.inf.br/index.php/article/download/50763. Acesso em: 28 abr. 2019.

ARAÚJO, R. F. Mídias sociais e comunicação científica: análise altmétrica em artigos de periódicos da ciência da informação. Informação \& Informação, Londrina, v. 21, n. 2, 2015. Disponível em: http://dx.doi.org/10.5433/1981-8920.2016v21n2p68. Acesso em: Acesso em: 10 jan. 2020.

BORBA, V. R.; ALVAREZ, G. R.; CAREGNATO, S. E. Análise Altmétrica da Produção Científica das Revistas brasileiras em Ciência da Informação Qualis A1 (2011-2017) no Mendeley. Encontros Bibli: revista eletrônica de biblioteconomia e ciência da informação, Florianópolis, v. 24, n. 55, p. 1-20, maio 2019. Disponível em: https://periodicos.ufsc.br/index.php/eb/article/view/1518-2924.2019.e58658/39972. Acesso em: 30 jan. 2020.

COSTA, B.; OLIVEIRA, M.; ARAÚJO, R. F. Impactos das teses e dissertações do Programa de Pós-Graduação em Ciência da Informação da UFMG. Informação em Pauta, v. 4, n. 2, p. 11-31, 27 dez. 2019. Disponível em: http://www.periodicos.ufc.br/informacaoempauta/article/view/42444. Acesso em: 10 fev. 2020. 
COORDENAÇÃO DE APERFEIÇOAMENTO DE PESSOAL DE NÍVEL

SUPERIOR. Plataforma Sucupira: Qualis periódico. Brasília, 2020. Disponível em: https://sucupira.capes.gov.br/sucupira/public/consultas/coleta/veiculoPublicacaoQualis/1 istaConsultaGeralPeriodicos.jsf. Acesso em: 30 jan. 2020.

GOUVEIA, F. C. A altmetria e a interface entre a ciência e a sociedade. Trabalho, Educação e Saúde, Rio de janeiro, v. 14, n. 3, p. 643-645, 2016. Disponível em: https://dx.doi.org/10.1590/1981-7746-sip00126. Acesso em: 30 jan. 2020.

GOUVEIA, F. C. Estudos altmétricos no Brasil: uma análise a partir dos currículos da Plataforma Lattes-CNPq. Transinformação, v. 31, e190027, 2019. Disponível em: http://dx.doi.org/10.1590/2318-0889201931e190027. Acesso em: 30 jan. 2020.

HAYON, S.; TRIPATHI, H.; STORMONT , I. M. Twitter mentions and academic citations in the urology literature. Urology, New York, v. 123, p. 28-33, 2019.

Disponível em: https://doi.org/10.1016/j.urology.2018.08.041. Acesso em: 30 jan. 2020.

MILOJEVIĆ, S.; SUGIMOTO, C. R. Introduction metrics \& ASIS\&T. Bulletin of the American Society for Information Science \& Technology, Silver Spring, v. 38, n. 6, p. 9-11, 2012. Disponível em: https://doi.org/10.1002/bult.2012.1720380604. Acesso em: 12 abr. 2019.

OLIVEIRA, E. da C. P. de. Comunicação científica e redes sociais. In: PINHEIRO, L. V. R.; E. da C. P. de O. (Orgs.). Múltiplas facetas da comunicação e divulgação científicas: transformações em cinco séculos. Brasília: IBICT, 2012. 367 p.

OLIVEIRA, T. M. de. As métricas alternativas e Ciência Aberta na América Latina: desafios para a democratização do conhecimento. Transinformação, Campinas, v. 31, e190089, 2019. Disponível em:

http://www.scielo.br/scielo.php?script=sci_arttext\&pid=S0103-37862019000100200\&1 ng=en\&nrm=iso. Acesso em: 30 jan. 2020 .

PRIEM, J. et al. Altmetrics: a manifesto. [S.l: s.n.]. 2010. Disponível em: http://altmetrics.org/manifesto/. Acesso em: 30 jan. 2020.

ROSENKRANTZ, A. B.; AYOOLA, A.; SINGH, K. Alternative metrics (“Altmetrics") for assessing article impact in popular general radiology journals. Academic Radiology, v. 24, n. 7, p. 891-897, July 2017. Disponível em: https://doi.org/10.1016/j.acra.2016.11.019. Acesso em: 28 abr. 2018.

SANTOS, V. S. dos. Animais fistulados. Brasil Escola. Disponível em: https://brasilescola.uol.com.br/biologia/animais-fistulados.htm. Acesso em: 30 jan. 2020.

SCHWARTZ, B. W. A. et al. Maximizing versus satisficing: happiness is a matter of choice. Journal of Personality and Social Psychology, Washington, v. 83, p. 1178-1197, 2012. Disponível em: http://dx.doi.org/10.1037/0022-3514.83.5.1178. Acesso em: 30 jan. 2020. 
SCHNITZLER, K. et al. Using TwitterTM to drive research impact: A discussion of strategies, opportunities and challenges. International Journal of Nursing Studies, Elmsford, v. 59, p. 15- 26, July 2016. Disponível em: 10.1016 / j.ijnurstu.2016.02.004. Acesso em: 30 jan. 2020.

SCIELO ANALYTICS. Ciência e Agrotecnologia: collection composition. São Paulo, 2019. Disponível em: https://analytics.scielo.org/w/publication/size. Acesso em: 30 jan. 2020 .

SHEKHAWAT, K. S.; CHAUHAN, A. Altmetrics: a new paradigm for scholarly communication. Indian Journal of Dental Research, Nova Delhi, v. 30, n. 1, p. 125-126, 2019. Disponível em:

http://www.ijdr.in/article.asp?issn=0970-9290;year=2019; volume $=30 ;$ issue $=1 ;$ spage $=12$ 5; epage $=126$; aulast=Shekhawat. Acesso em: 30 jan. 2020.

\section{UNIVERSIDADE FEDERAL DE LAVRAS. Editora UFLA. Ciência e} Agrotecnologia. Lavras, 2020. Disponível em: http://www.editora.ufla.br/index.php/revistas/ciencia-e-agrotecnologia. Disponível em: 30 jan. 2020.

VARELLA, F. Mídias sociais e altmetria: o impacto da produção científica em novas métricas. Esboços: histórias em contextos globais, Florianópolis, v. 26, n. 42, p. 224-228, jul. 2019. ISSN 2175-7976. Disponível em: https://periodicos.ufsc.br/index.php/esbocos/article/view/2175-7976.2019v26n42p224/4 0327. Acesso em: 30 jan. 2020.

VRABEL, M. Beyond the Impact Factor. Oncology Nursing Forum, Pittsburgh, v. 46, n. 2, p. 143-145, 2019. Disponível em:

https://onf.ons.org/onf/46/2/beyond-impact-factor. Acesso em: 30 jan. 2020.

WOOLDRIDGE, J.; KING, M. B. Altmetric scores: An early indicator of research impact. Journal of the Association for Science and Information Technology, New York, v. 70, n. 3, n. 271-282, mar. 2019. Disponível em: https://doi.org/10.1002/asi.24122. Acesso em: 30 jan. 2020.

ZHENG, H. et al. Social media presence of scholarly journals. The Journal of the Association for Information Science and Technology, New York, v. 70, n. 3, p. 256-270, mar. 2019. Disponível em: https://doi.org/10.1002/asi.24124. Acesso em: 23 abr. 2019. 